\title{
Design of Transmitter and Receiver Architectures for Differential Amplitude Pulse-Position Modulation Scheme
}

\author{
Mehmet Sönmez \\ *Electrical and Electronics Engineering, Osmaniye Korkut Ata University \\ DOI: 10.29322/IJSRP.11.12.2021.p12070 \\ http://dx.doi.org/10.29322/IJSRP.11.12.2021.p12070
}

\begin{abstract}
In this paper, a FPGA (Field Programmable Gate Arrays) based modulator is proposed for DAPPM (Differential Amplitude Pulse Position Modulation) technique. The proposed modulator is designed on FPGA complier Quartus program. Moreover, the simulation results are obtained on modelsim altera program. Addition to FPGA-based modulator, a demodulator is designed on FPGA complier to estimate the data bits at the receiver side. The proposed modulator and demodulator schemes are successfully implemented according to simulation results which are obtained from modelsim altera program. In addition, variable threshold values are considered to detect the data bits on the receiving side. Slot error rate (SER) performances of demodulators used variable threshold values were analyzed in the simulation environment. An optimum threshold value was estimated according to the analysis results.
\end{abstract}

Index Terms- DAPPM-FPGA-Transceiver Design-VLC

\section{INTRODUCTION}

$\mathrm{T}$ he Visible Light Communication Systems (VLC) is one of the Optical Wireless Communication (OWC) methods. Nowadays, it can be argued that VLC has very inefficient performance for long distance between transmitter and receiver. Hence, it can be applied on short transmission distance [1]. VLC systems provide the data transmission both using a LED (Light Emitted Diode) at transmitter side and using a photodiode at the receiver side [2]. The low frequency response of photodiode can cause that VLC supports the low data rate. Addition to this, it can cause the low BER (Bit Error Rate) performance and slot interference for VLC systems $[3,4]$

It has been proposed the many modulation schemes for VLC systems in the literature. The one of the most basic schemes is OOK (On-Off Keying) technique that ensures the switching of LED against to data bit condition. Compared to another schemes considered position of filled slot, the ambient light reduces the BER performance of OOK due to fact that OOK uses a threshold value to detect the data bits. Addition to this, an adaptive threshold estimator must be used to detect data bits for OOK systems due to implementing of mobile systems [5, 6]. The PPM (Pulse Position Method) scheme is one of the most used modulation techniques since it doesn't use any threshold value to detect data bits. Two dimming methods have been improved to adjust the duty period of OOK and PPM: VOOK (Variable OOK) and VPPM (Variable PPM) schemes [7]. Thanks to variable methods, it can be adjusted the target dimming level of PPM and OOK methods.

In the literature, DPPM (Differential Pulse Position Modulation) scheme is proposed to reduce the number of transmitted slot of PPM signal. The DPPM method uses one filled slot and a few or zero empty slots with respect to the decimal value of transmitting data symbol [8]. It is investigated the DPPM method with respect to bandwidth efficiency and power consumption. Compared to PPM method, it is clear that DPPM method gives very effective results in terms of these parameters. In order to increase the bandwidth efficiency and power consumption of DPPM, it has been proposed the DAPPM by authors of [9]. When investigate the analysis results in the literature, it is clear that DAPPM supports higher data rate than that of PPM and DPPM [10].

As we known, it can be argued that there is not any digital based system to apply the DAPPM method in the literature. Therefore, in this paper a transmitter model has been improved to implement the FPGA board. It is observed from modelsim-altera simulation results that the proposed transmitter system successfully generates the DAPPM signal by taking account data bits. Addition to this, a DAPPM demodulator scheme has been operated to detect data symbol from received DAPPM signal. In addition, it is shown that it is important the selection of an optimum threshold value. It is investigated the SER performance of system under variable threshold value effect. 


\section{MATERIALS AND METHODS}

In the literature, there are many modulation schemes to apply on optical communication systems. The most basic of these methods can be given as PPM and OOK schemes. Another transmission scheme can be referred to as DPPM which has been proposed to increase the bandwidth efficiency of PPM scheme. The authors of (Sethakaset ve Gulliver, 2005) modified DPPM scheme by using two amplitude levels. The modified DPPM scheme is called DAPPM technique. In Table-I, codewords are given with respect to data symbols.

In the table, MPPM and SPPM are defined as M-level PPM and Shortened PPM schemes. Thanks to proposed schemes, it is aim to decrease the slot number of PPM. This case will result in an increase the bandwidth efficiency of PPM scheme. The A value for DAPPM given in table gets 1 or 2 . The $\mathrm{L}$ can be increased to 4 as shown in the table. Therefore, the $\mathrm{L}$ and $\mathrm{A}$ values for the DAPPM given in the table are 4 and 2 , respectively.

Table-I. The codewords for modulation schemes

\begin{tabular}{|l|l|l|l|l|l|l|}
\hline OOK & $8-P P M$ & DPPM & DAPPM & $\left(\frac{5}{2}\right)$ MPPM & $\begin{array}{l}\text { Offset } \\
\text { PPM }\end{array}$ & SPPM \\
\hline 000 & 00000001 & 1 & 1 & 11000 & 0000 & 00001 \\
\hline 001 & 00000010 & 01 & 01 & 10100 & 0001 & 00010 \\
\hline 010 & 00000100 & 001 & 001 & 10010 & 0010 & 00100 \\
\hline 011 & 00001000 & 0001 & 0001 & 10001 & 0100 & 01000 \\
\hline 100 & 00010000 & 00001 & 2 & 01100 & 1000 & 10001 \\
\hline 101 & 00100000 & 000001 & 02 & 01010 & 1001 & 10010 \\
\hline 110 & 01000000 & 0000001 & 002 & 01001 & 1010 & 10100 \\
\hline 111 & 10000000 & 00000001 & 0002 & 00110 & 1100 & 11000 \\
\hline
\end{tabular}

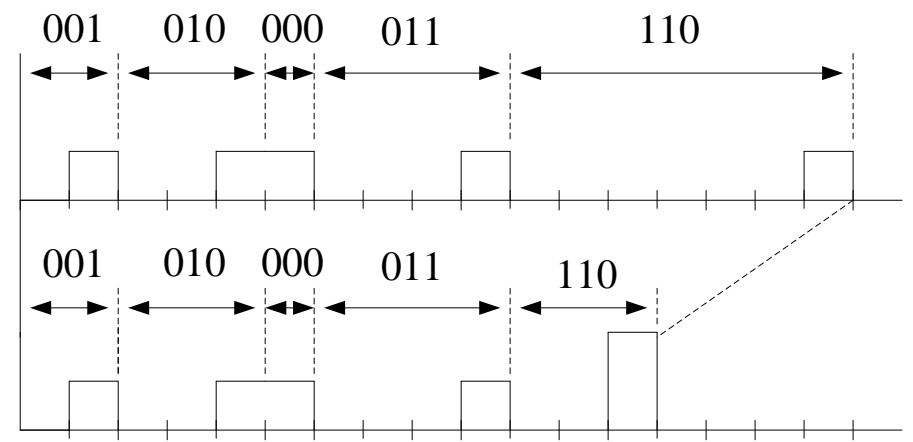

Fig. 1 The time-chart performance comparison of DAPPM and DPPM schemes

As shown in the Fig. 1, the decimal value of data symbol determines sequential empty slot number. A filled slot is generated after it is ensured the equality among decimal value and empty slot number. Additionally, the lengths of DPPM and DAPPM signal are equal to each other when data symbols get "001", "010", "000" and "011". However, the lengths of these schemes will not equal to each other as shown in the Table-I when the MSB (Most Significant Bit) is rising to logical '1'. In this case, instead of P power level DAPPM generates the modulated signal at the power of $2 \mathrm{P}$ level. The slot number is decreased by using two power levels. The case number of DAPPM can be given by

$$
\mathrm{M}=\log _{2}(\mathrm{AxL})
$$

where, M presents the number of encoded bits in a symbol. According to Table-I and Fig. 1, the number of bit that is included in one symbol is equal to 3. Therefore, $\mathrm{A}$ and $\mathrm{L}$ values get 2 and 4, respectively. However, the status number is equal to 8 since it can be presented as $2 \mathrm{M}$. The $\mathrm{M}$ value for DPPM can be expressed by

$$
\mathrm{M}=\log _{2}(\mathrm{~L})
$$

where, A gets 1 value since the amplitude level is equal to 1 for DPPM scheme. Therefore, it is shown from Table I that the L gets 8 for both DAPPM and DPPM. Addition to this, the M value is 3. According to this expression, it is claimed that the DAPPM decreases slot number of DPPM by using two power levels. If Ts can be expressed as slot duration, the integral value of one slot of DAPPM can be given as follows:

$$
I(t)=\int_{n T s}^{(n+1) T s} s(t) d t
$$


where, $\mathrm{I}(\mathrm{t})$ and $\mathrm{s}(\mathrm{t})$ are introduced as output of integrator and received signal, respectively. If the first slot is pointed out zeroth slot, the $\mathrm{n}$ value in the equation observes the slot order. The integral value is compared with two power levels to detect slot level. One of threshold values is used to detect the MSB bit as zero or one, and the other decides whether the level of the slot is logical '1' or ' 0 '. This expressing given by,

$$
\left\{\begin{array}{ll}
\mathrm{I}(\mathrm{t})<\mathrm{th}_{1} & ; \mathrm{ds}=0 \\
\mathrm{th}_{1} \leq \mathrm{I}(\mathrm{t})<\mathrm{th}_{2} & ; \mathrm{ds}=1 \\
\mathrm{th}_{2} \leq \mathrm{I}(\mathrm{t}) & ; \mathrm{ds}=2
\end{array}\right\}
$$

where, ds can be expressed as slot level. If the threshold value (th1) is greater than the integral value I(t), the slot is at logical ' 0 ' level. If the $\mathrm{I}(\mathrm{t})$ value is between two threshold values in the second case, the slot is defined as logical ' 1 '. However, the MSB is logical ' 0 '. In other case, if the $\mathrm{I}(\mathrm{t})$ value is greater than the threshold value-2, MSB bit and slot is logical ' 11 '. The demodulation process is detailed by giving a block diagram of FPGA-based digital architecture.

In Fig. 2, the data block observes the transmitting symbol. The data encoder block groups the data signal by merging data bits of three. One of these bits determines the amplitude of the filled slot while others determine the number of empty slots. The accumulator output is initially zero in decimal. It is increased until it equals the decimal value of data bits at the other input of the comparator. As soon as values at the comparator input are equal, the signal at the comparator output rises to the logical '1' level. This signal is applied on accumulator to reset this block. When the values at the comparator input are different, the comparator output is at the logical ' 0 ' level. The amplitude of filled slot is only equal to $2 \mathrm{P}$ when output of comparator and other data signal is logical ' 1 '.

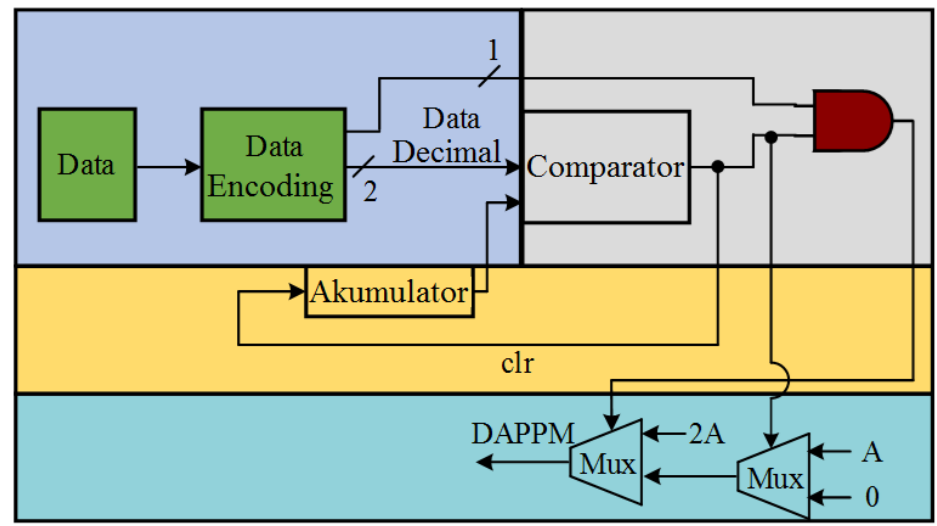

Fig. 2 Proposed FPGA-based DAPPM transmitter scheme

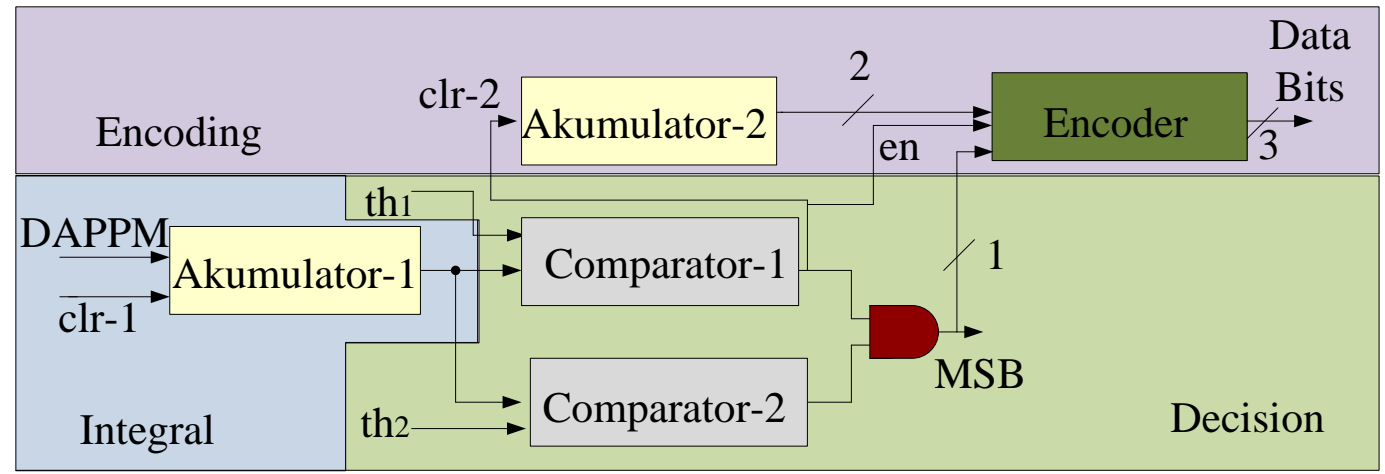

Fig. 3. The proposed FPGA-based DAPPM receiver system

Receiver unit block diagram is shown in Fig. 3. The Accumulator-1 block given in the figure performs the integral operation that is given in Equation (3). The criteria's given in Equation (4) is valid for decision stage. The integral output is passed through two different comparators and the decision processes given in equation 4 are applied. If both comparators are at the logical '1' level, it is assigned the MSB to the logical '1' level, otherwise the MSB value gets '0'. The accumulator block is reset by using output signal of comparator when this signal becomes logical '1'. In other stage, the encoder block merges MSB with accumulator- 2 block by using en signal.

\section{Simulation Results}

In this section, the simulation results obtained in the modelim-altera program are given for proposed transmitter and receiver architectures. The first simulation is employed for the transmitting system. According to simulation results, it is observed that the 
DAPPM signal is successfully generated with respect to data symbols. The Fig. 4 shows the modelsim-Altera simulation results for transmitter system.

Data, accumulator, D.decimal and D.Encoder parameters defined in simulation results observe the outputs of the blocks used in the block diagram given in Fig. 2. As can be seen from the figure, the data decimal indicates the decimal value of the last two bits of the information bits that are meaningless to be transmitted. It can be obtained the last two bits of the information bits at output of D.Encoder. For example, while the first transmitted symbol is "110", the most significant bit is '1' and the last two non-significant bits are seen as "10". It is seen that the decimal value of the meaningless bits is also 2. As observed in the figure, the accumulator counts up to the data decimal value. Additionally, the DAPPM signal has logical '1' level when the data decimal and the values of the accumulator are equal to each other.

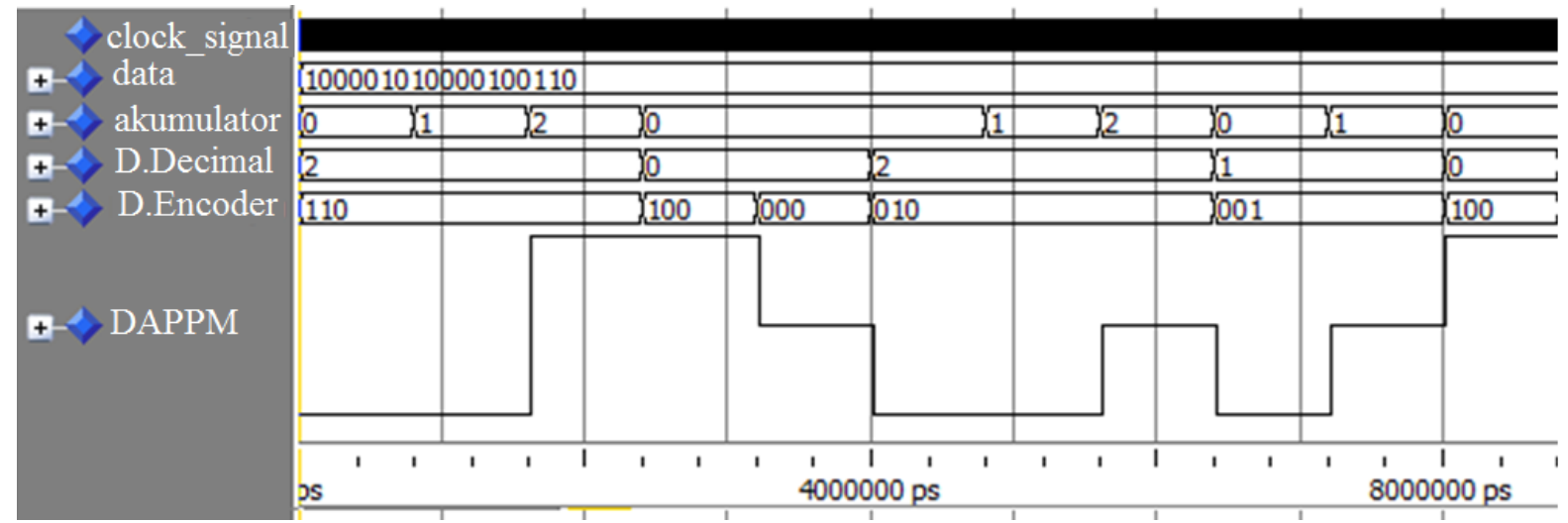

Fig. 4. The simulation results of transmitter system

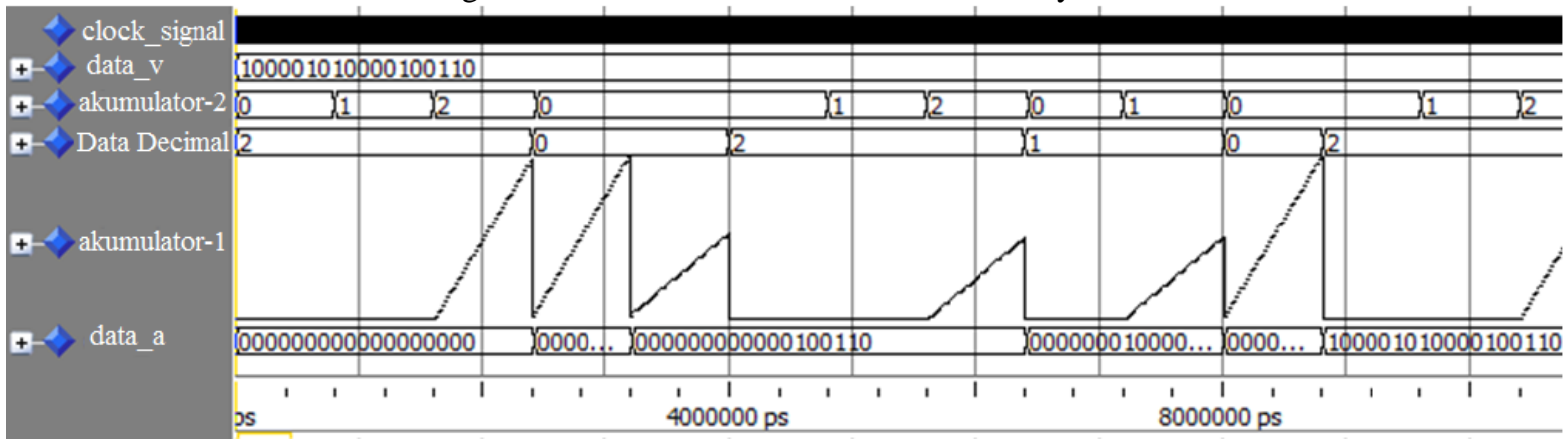

Fig. 5. The simulation results of transceiver system

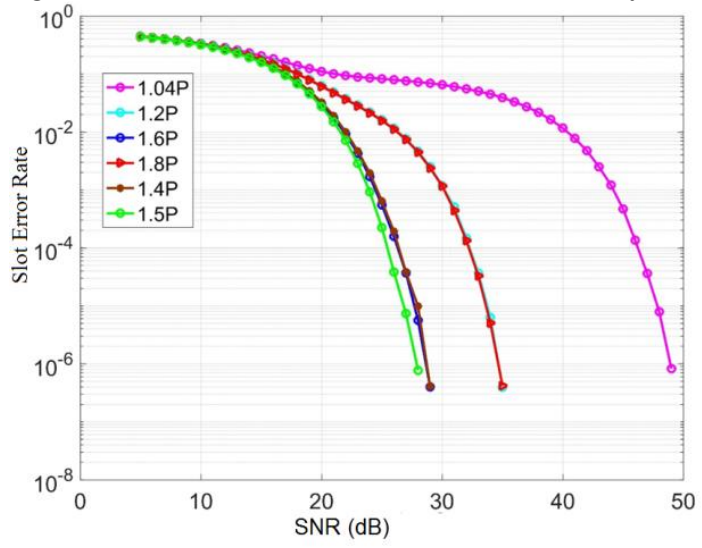

Fig. 6. The simulation results of variable threshold values

The Fig. 5 shows the modelsim simulation results of receiver system. The parameters given in the simulation results are defined by considering the parameters used in the block diagram given in Fig. 3. As shown in the figure, accumulator-2 counts up to the data decimal value. Therefore, two meaningless bits are obtained by taking account the accumulator result. If the result of the accumulator -1 is carefully examined, it generates 3 different values by providing the expression given in the Eq (4). As seen in the figure, when the MSB value is '1', the integral output reaches to its maximum value. It is aim to investigate the effect of optimum threshold value in the paper. Hence, it is given the simulation results observed in Fig. 6. 
According to the simulation results, it is thought that the logical '1' level of the MSB bit is sent as $2 \mathrm{P}$ and the logical '0' level is sent as P. According to simulation results, the slot error rate performance is gradually decreased while the distance between optimum threshold value is increased

\section{CONCLUSION}

In the study, a digital circuit-based modulator and demodulator design is proposed for the DAPPM method. The proposed architectures are designed in the FPGA compiler Quartus program. The operation of the transceiver architectures was followed on the simulation results obtained from the Modelsim-Altera program. In addition, it is shown that it is important the selection of an optimum threshold value. It is investigated the SER performance of system under variable threshold value effect. According to simulation results, adaptive threshold methods are very effective under mobile user systems. In future, it may be investigated slot synchronization problem of DAPPM and DPPM methods. This issue is very important to overcome the measurement of bit error rate performances of these modulation schemes.

\section{REFERENCES}

[1] Guo, M., Bai, Z., Pang, K., Hao, X., Tian, J., Kwak, K., 2020. Design and Analysis of Color Shift Keying Modulation based Cooperative SM VLC System. 2020 International Wireless Communications and Mobile Computing (IWCMC), Limassol, Cyprus, June 2020.

[2] Shi, J., He, J., Jiang, Z., Chang, G.K., 2020. Modulation Format Shifting Scheme for Optical Camera Communication. IEEE Photonics Technology Letters, $32: 1167-1170$.

[3] Wu, J.T., Chow, C.-W., Liu, Y., Hsu, C.W., Yeh, C.H., 2017. Performance enhancement technique of visible light communications using passive photovoltaic cell. Optics Communications, 392:119-122.

[4] Hosney, M., Selmy, H. A. I., Srivastava, A., Elsayed, K. M. F., 2020. Interference Mitigation Using Angular Diversity Receiver With Efficient Channel Estimation in MIMO VLC. IEEE Access, 8: 54060-54073.

[5] Amini, C., Taherpour, A., 2017. Adaptive threshold device for detection of reflections based visible light communication. Optics Communications, 388 : 1-11.

[6] Li, S., Pandharipande, A., Willems, F. M. J., 2017. Adaptive visible light communication LED receiver. 2017 IEEE SENSORS, Glasgow, Scotland, Nov. 2017.

[7] Lee, K., Park, H., 2011. Modulations for Visible Light Communications With Dimming Control. IEEE Photonics Technology Letters, 23:1136-1138.

[8] Zwillinger, D., 1988. Differential PPM has a higher throughput than PPM for the band-limited and average-power-limited optical channel. IEEE Transactions on Information Theory, 34: 1269-1273.

[9] Sethakaset, U., Gulliver, T. A., 2005. Differential Amplitude Pulse-Position Modulation for Indoor Wireless Optical Communications. EURASIP Journal on Wireless Communications and Networking, 2005: 3-11.

[10] Sethakaset, U., Gulliver, T. A., 2006. Channel Capacity of Differential Amplitude Pulse-Position Modulation (DAPPM) over Indoor Optical Wireless Communications. 2006 Asia-Pacific Conference on Communications, Busan, South Korea, December 2006.

\section{AUTHORS}

First Author - Mehmet Sönmez, Osmaniye Korkut Ata Univesity, mehmetsonmez@osmaniye.edu.tr Correspondence Author - Mehmet Sönmez, Osmaniye Korkut Ata Univesity, mehmetsonmez@ osmaniye.edu.tr 